\title{
Impacto de la revascularización y factores asociados en el salvamento de la extremidad en pacientes con pie diabético
}

Carlos A. Hinojosa, Estefanía Boyer-Duck, Javier E. Anaya-Ayala, Ana E. Núñez-Salgado, Hugo Laparra-Escareno y René Lizola

Instituto Nacional de Ciencias Médicas y Nutrición Salvador Zubirán, Dirección de Cirugía, Sección de Angiología y Cirugía Vascular. Ciudad de México, México

\section{Resumen}

Introducción: El pie diabético es causa frecuente de hospitalización. Objetivo: Examinar el impacto de la revascularización en la preservación de los miembros inferiores. Método: Estudio retrospectivo de pacientes diabéticos con úlceras en el pie. Se evaluó extensión del tejido perdido conforme las clasificaciones PEDIS y de Wagner, así como indicaciones y técnicas de revascularización. Los factores involucrados en la amputación mayor y el salvamento de la extremidad fueron evaluados por pruebas Fisher y chi cuadrado. Resultados: Fueron estudiados 307 pacientes con edad media de 61 años; 198 (64 \%) eran hombres; 53 (17\%) fueron sometidos a revascularización de la extremidad, 26 (8 \%) con técnicas endovasculares y 27 (9 \%) por cirugía abierta; 27 categorizaron en el grado 3 de la clasificación PEDIS (51\%) y 21 (41 \%) en el 4 de Wagner; 52 \% de los pacientes revascularizados necesitó amputación mayor versus $25 \%$ de los no revascularizados. Las comorbilidades, variables demográficas, complicaciones y mortalidad no mostraron diferencias cuando se compararon quienes necesitaron una amputación mayor y los que no. Conclusión: Menos de 50 \% de los pacientes salvó la extremidad a pesar de la revascularización. La referencia temprana a cirugía vascular y los criterios de selección apropiados podrían incrementar el salvamento de extremidades.

PALABRAS CLAVE: Pie diabético. Salvamento de extremidad. Amputación.

\begin{abstract}
Introduction: Diabetic foot is a common cause of hospitalization. Objective: To examine the impact of revascularization on lower limb salvage. Method: Retrospective study of diabetic patients with foot ulcers. The extent of tissue loss was assessed according to the PEDIS and Wagner classifications, and revascularization indications and techniques were evaluated. Factors involved with major amputation and limb salvage were assessed with Fisher's and chi-square tests. Results: $A$ total of 307 patients with a mean age of 61 years were included in the study; 198 (64\%) were males; 53 (17\%) underwent limb revascularization, 26 (8\%) with endovascular techniques and 27 (9\%) with open surgery; 27 belonged to PEDIS grade 3 (51\%) and $21(41 \%)$ to Wagner's classification grade 4; 52\% of revascularized patients required major amputation versus $25 \%$ of those without revascularization. Comorbidities, demographic variables, complications and mortality showed no differences when patients who required major amputation were compared with those who didn't. Conclusion: Despite revascularization, the limb was preserved in less than $50 \%$ of patients. Early referral to vascular surgery and appropriate patient-selection criteria might increase limb salvage.
\end{abstract}

KEY WORDS: Diabetic foot. Limb salvage. Amputation.

Fecha de recepción: 19-07-2016

Fecha de aceptación: 30-12-2016

DOI://dx.doi.org/10.24875/GMM.18002772
Gac Med Mex. 2018;154:190-197

Disponible en PubMed www.gacetamedicademexico.com 


\section{Introducción}

Las lesiones ulcerosas en las extremidades inferiores de pacientes con diabetes mellitus son una complicación con consecuencias importantes. ${ }^{1}$ Se ha estimado que el riesgo de desarrollar una úlcera al menos una vez en la vida es de aproximadamente $25 \%$ en este grupo de pacientes. ${ }^{1}$ Debido a que estas lesiones están asociadas con riesgo aumentado de amputación de la extremidad afectada, el objetivo terapéutico primario es la preservación del miembro. ${ }^{2}$ Desde el punto de vista económico se estima que hasta una tercera parte del costo del tratamiento en los pacientes diabéticos es atribuible al manejo de úlceras en el pie. ${ }^{3}$ Estas lesiones se desarrollan en aproximadamente un cuarto de los pacientes con diabetes y en la mitad de ellos puede ocurrir infección seria de tejidos que requiere hospitalización, uso de antibióticos intravenosos y tratamiento quirúrgico agresivo; a pesar de un tratamiento óptimo, hasta una quinta parte de estos pacientes termina necesitando una amputación mayor. ${ }^{4}$ Una vez que una amputación mayor por pie diabético ha ocurrido, la mortalidad excede a la reportada para algunos tipos de cáncer: oscila entre 39 a $80 \%$ en los primeros cinco años. ${ }^{5,6}$ Para reducir el número de amputaciones es esencial una coordinación del sistema de salud y un abordaje multidisciplinario. ${ }^{7,8}$

El desarrollo de úlceras en el pie es atribuible a numerosos factores que interactúan, entre los cuales destacan la neuropatía periférica y la enfermedad arterial periférica. ${ }^{7}$ Esta última es un factor que tiene un impacto considerable, ya que hasta en $50 \%$ de los pacientes se puede demostrar un trastorno en la circulación. ${ }^{9}$ La revascularización de la extremidad, ya sea por cirugía abierta (que incluye la reconstrucción arterial directa o la derivación) o procedimientos endovasculares, es el tratamiento invasivo disponible para mejorar los síntomas. ${ }^{10}$ Entre los pacientes diabéticos y no diabéticos con enfermedad arterial periférica, los investigadores clínicos han enfocado esfuerzos para entender el impacto de la revascularización endovascular. Ciertamente se ha demostrado reducción en la tasa de amputaciones y las complicaciones asociadas con la cirugía abierta, aunque algunos análisis empíricos no han reportado cambios significativos en la necesidad de amputaciones mayores, a pesar de técnicas menos invasivas de revascularización. ${ }^{11}$
Son escasas las investigaciones en México que han abordado el impacto directo de la revascularización y la tasa de cicatrización de úlceras como un resultado específico de la intervención en pacientes con pie diabético. ${ }^{12}$ El objetivo de este estudio fue evaluar el impacto de la revascularización abierta y endovascular en pacientes diabéticos con lesiones ulcerosas en el pie tratados en el Instituto Nacional de Ciencias Médicas y Nutrición Salvador Zubirán y determinar los factores que influyeron en la selección y el salvamento de la extremidad en esta población hasta antes de 2012 en esa institución.

\section{Método}

Estudio longitudinal, descriptivo y análisis retrospectivo que comprendió el periodo de 1997 a 2012, en el que se incluyeron 307 pacientes con diagnóstico confirmado de diabetes tipos 1 y 2 que desarrollaron lesión ulcerosa en el pie.

Conforme a la Organización Mundial de la Salud, se definió como "pie diabético" a la ulceración, infección y destrucción de tejidos de la extremidad inferior asociada con alteraciones neurológicas y diversos grados de enfermedad vascular periférica. ${ }^{13}$ La clasificaciones de Wagner y PEDIS - que incluye variables como el nivel perfusión, extensión, profundidad, pérdida de tejido, infección y neuropatía diabética- ${ }^{14}$ y fueron utilizadas para seleccionar a los pacientes que necesitaban algún procedimiento de revascularización.

Se recolectó información de variables demográficas (sexo, edad, nivel socioeconómico), comorbilidades, estudios de laboratorio (hemoglobina glucosilada, niveles de colesterol y triglicéridos), características de las úlceras (número, localización y profundidad), así como la presencia de osteomielitis.

Las intervenciones encaminadas al restablecimiento o mejoramiento de la circulación incluyeron las cirugías abiertas (derivación femoropoplítea, endarterectomía femoral, trombectomía) y las intervenciones endovasculares (angioplastia y colocación de endoprótesis vasculares); se analizaron las complicaciones y las reintervenciones. El desenlace se determinó con base en la necesidad de una amputación mayor (supracondílea o infracondílea). Por "salvamento de extremidad" se definió a la situación en la que se logró la cicatrización completa de la úlcera o la necesidad de una amputación menor o conservadora (dedo, transmetatarsiana), que permitió una rápida recuperación y 
rehabilitación en un periodo de tres meses. El análisis estadístico fue descriptivo, las variables clínicas y comorbilidades fueron expresadas en percentiles, media, mediana y desviación estándar. El análisis de los factores involucrados en el desenlace (amputación mayor contra salvamento de extremidad) fueron evaluados con pruebas de Fisher y chi cuadrado, con el objetivo de encontrar diferencias en los grupos. Las pruebas fueron realizadas con el programa estadístico Stata versión 14.0.

\section{Resultados}

Fueron incluidos 307 pacientes diabéticos con edad de $61 \pm 14$ años (media $\pm \mathrm{DE}$ ), valorados en la Clínica de Heridas del Instituto Nacional de Ciencias Médicas y Nutrición Salvador Zubirán con diagnóstico de pie diabético; 198 (64\%) pertenecían al sexo masculino y 109 (36\%) al femenino (Tabla 1).

Tenían diagnóstico de diabetes tipo 2, 297 pacientes $(97 \%)$ y $10(3 \%)$ diabetes tipo 1; 226 pacientes $(74 \%)$ presentaban hipertensión arterial sistémica, 200 (65\%) dislipidemias, 114 (37\%) diagnóstico conocido de enfermedad arterial periférica y 54 (18\%) historia de infarto agudo de miocardio; 53 (17\%) fueron sometidos a intervención de revascularización, 26 por técnicas endovasculares (angioplastia o colocación de endoprótesis vasculares) y 27 por revascularización abierta. La decisión de intervenir se fundamentó en las clasificaciones PEDIS y de Wagner, con el objetivo de evitar una amputación mayor. Clasificaron en el grado 3 de PEDIS, 27 pacientes (51\%) y $21(41 \%)$ en el grado 4 de la de Wagner (Figura 1); $52 \%$ de los pacientes revascularizados necesitó amputación mayor en comparación con $25 \%$ de los no revascularizados.

Las Tablas 2 y 3 y la figura 2 incluyen las variables demográficas y comorbilidades en los pacientes revascularizados y no revascularizados, que no mostraron diferencias cuando se compararon los pacientes

Tabla 1. Distribución de acuerdo con el sexo, de los pacientes que salvaron la extremidad o fueron sometidos a amputación mayor

\begin{tabular}{lllll}
\hline & $\begin{array}{c}\text { Salvamento de extremidad* } \\
(\mathbf{\%})\end{array}$ & $\begin{array}{c}\text { Amputación } \\
\text { mayor } \\
(\%)\end{array}$ & $\begin{array}{c}\text { Total } \\
(\mathbf{n})\end{array}$ & $\mathbf{p}$ \\
\hline Sexo & Mujeres: 62 & Mujeres: 38 & 198 & \\
& Hombres: 63 & Hombres: 37 & 109 & 0.80 \\
\hline *Incluye amputación menor & & &
\end{tabular}

que necesitaron amputación mayor con los pacientes revascularizados que requirieron amputación conservadora. En los pacientes no revascularizados, el diagnóstico de enfermedad arterial periférica $(p=0.001)$ e historia de infarto agudo de miocardio $(p=0.003)$ influyeron en la necesidad de realizar una amputación mayor.

Por otra parte, en el grupo de pacientes que no recibió revascularización se observó que los antiagregantes plaquetarios contribuyeron en el salvamento de la extremidad $(p=0.001)$. La edad media de los pacientes que requirieron amputación mayor fue de 65 años contra 58 años de los no amputados ( $p=0.0001)$; en los pacientes no revascularizados que necesitaron amputación mayor la edad media fue de 63 años comparada con 71 años de quienes recibieron revascularización $(p=0.006)$ No hubo diferencia en mortalidad y complicaciones mayores en los pacientes revascularizados que salvaron la extremidad o requirieron amputación mayor. La Figura 2 representa la distribución por edad de los pacientes sometidos o no a revascularización, la presencia de osteomielitis y el desenlace (amputación conservadora de pie, salvamento de extremidad o amputación mayor).

Las amputaciones mayores ocurrieron más en los pacientes con grado 3 de la clasificación de PEDIS y 4 de Wagner. No se observó tendencia a mayor número de estas amputaciones, independientemente de la técnica de revascularización. Las Figuras 3 y 4 representan la distribución de los pacientes con amputación mayor que no recibieron revascularización y de quienes se sometieron a revascularización, endovascular o abierta, de acuerdo con las clasificaciones PEDIS y de Wagner.

\section{Discusión}

La "isquemia crítica de la extremidad" es un concepto introducido en 1982; desde entonces sus diferentes clasificaciones han marcado la pauta en la decisión clínica para realizar procedimientos de revascularización. ${ }^{15}$ Originalmente definía a pacientes sin diabetes que tenían mayor riesgo de perder la extremidad pon isquemia crónica; los autores que acuñaron el concepto coincidían en que los pacientes con diabetes tenían una expresión clínica más compleja que incluía neuropatía, sepsis e isquemia. En los años siguientes, el uso de "isquemia crítica" fue atribuido inapropiadamente a un espectro más amplio de 

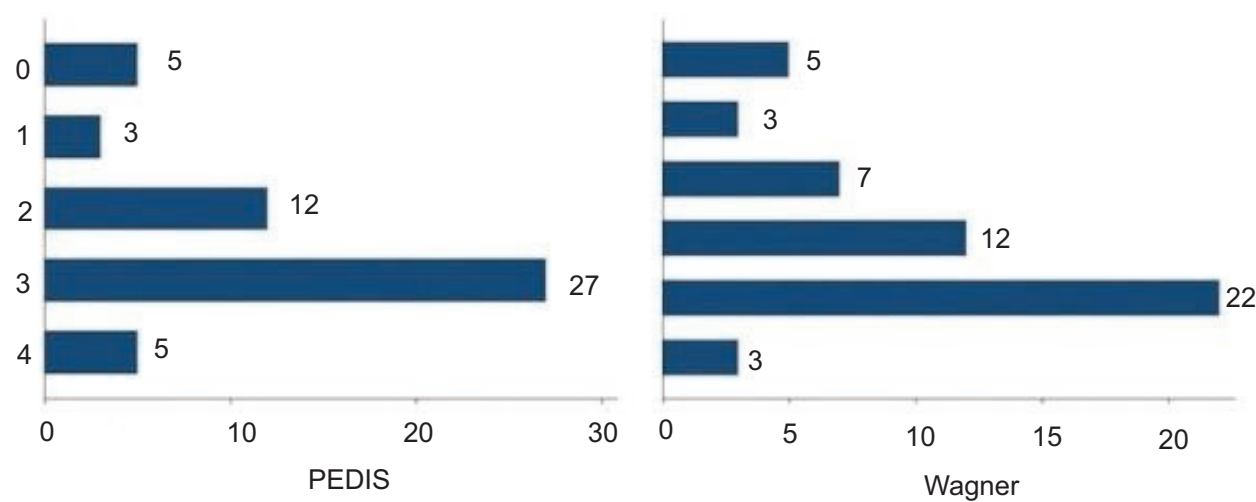

Figura 1. Distribución del número de pacientes sometidos a revascularización de acuerdo con las clasificaciones PEDIS y de Wagner.

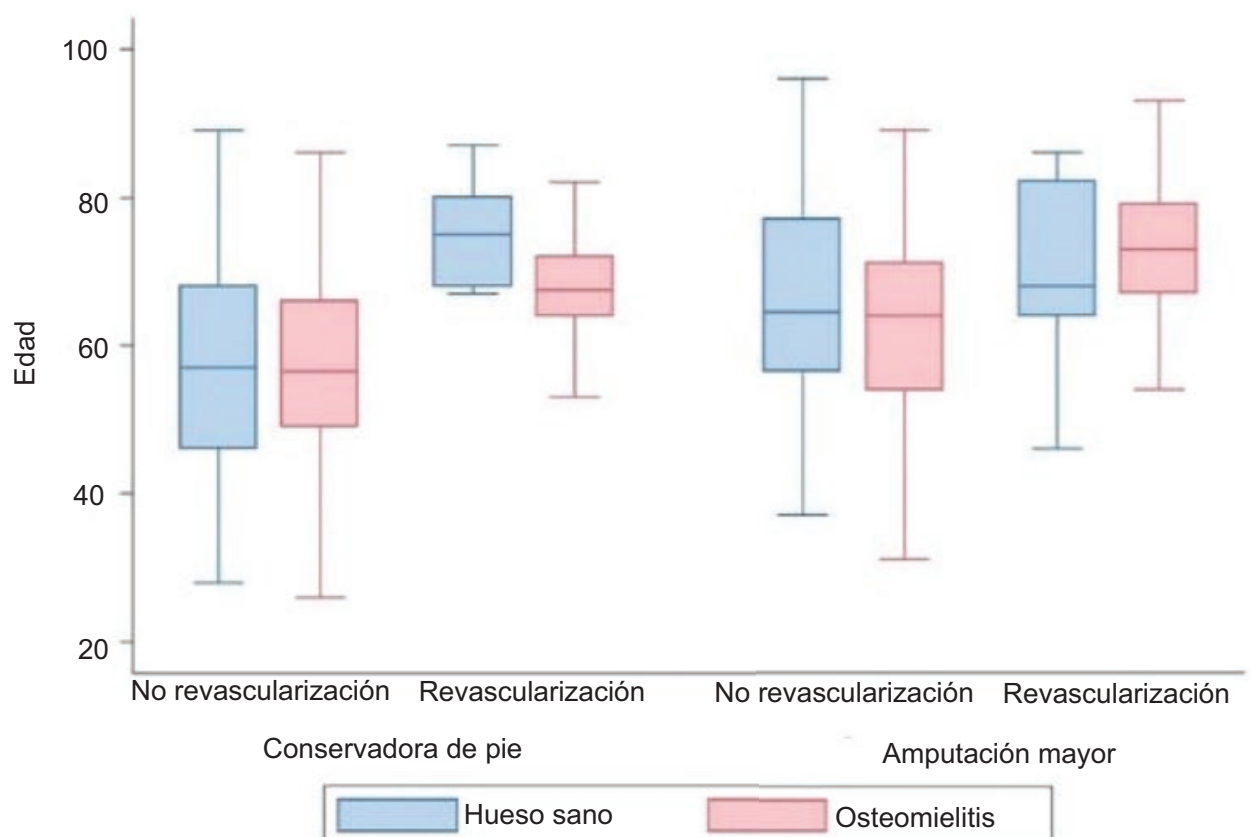

Figura 2. Distribución por edad de los pacientes sometidos o no a revascularización, la presencia de osteomielitis y el desenlace (amputación conservadora de pie, salvamento de extremidad o amputación mayor).

expresiones clínicas, sin tomar en consideración si se trataba de pacientes diabéticos o no diabéticos.

Por otro lado, las clasificaciones PEDIS y de Wagner se han utilizado para clasificar el grado de destrucción de tejidos e infección en pacientes diabéticos con lesiones ulcerosas, sin embargo, estos sistemas han fallado en proveer suficientes detalles en relación con el estado de perfusión y son sistemas que evalúan úlceras, sin mencionar la presencia gangrena, la cual se conoce que incrementa el grado de amputación en comparación con una úlcera. ${ }^{16}$ Aunque la clasificación de Wagner ${ }^{17}$ incluye gangrena, no diferencia claramente si el estado isquémico es resultado de infección o gangrena, además de no caracterizar el grado de infección, isquemia y extensión de las lesiones. En 2014, la Sociedad de Cirugía Vascular en
Estados Unidos de América introdujo la clasificación Wound, Ischemia, and foot Infection, con el objetivo de seleccionar más apropiadamente a los pacientes que requerían revascularización e incrementar el conocimiento de los pacientes que podrían beneficiarse de estas intervenciones. ${ }^{18}$ En años recientes, autores como Zhan ${ }^{19}$ han utilizado esta clasificación para evaluar correctamente la definición y correlacionar las intervenciones con el índice de cicatrización de heridas y el salvamento de extremidad. En su estudio, Zhan incluyó 201 pacientes, de ellos, 42 requirieron amputación mayor $(21 \%)$ y 159 (78 \%) salvaron su extremidad a un año, este grupo tenía una prevalencia de enfermedad más avanzada en comparación con el grupo de salvamento de extremidad (uno a tres). 


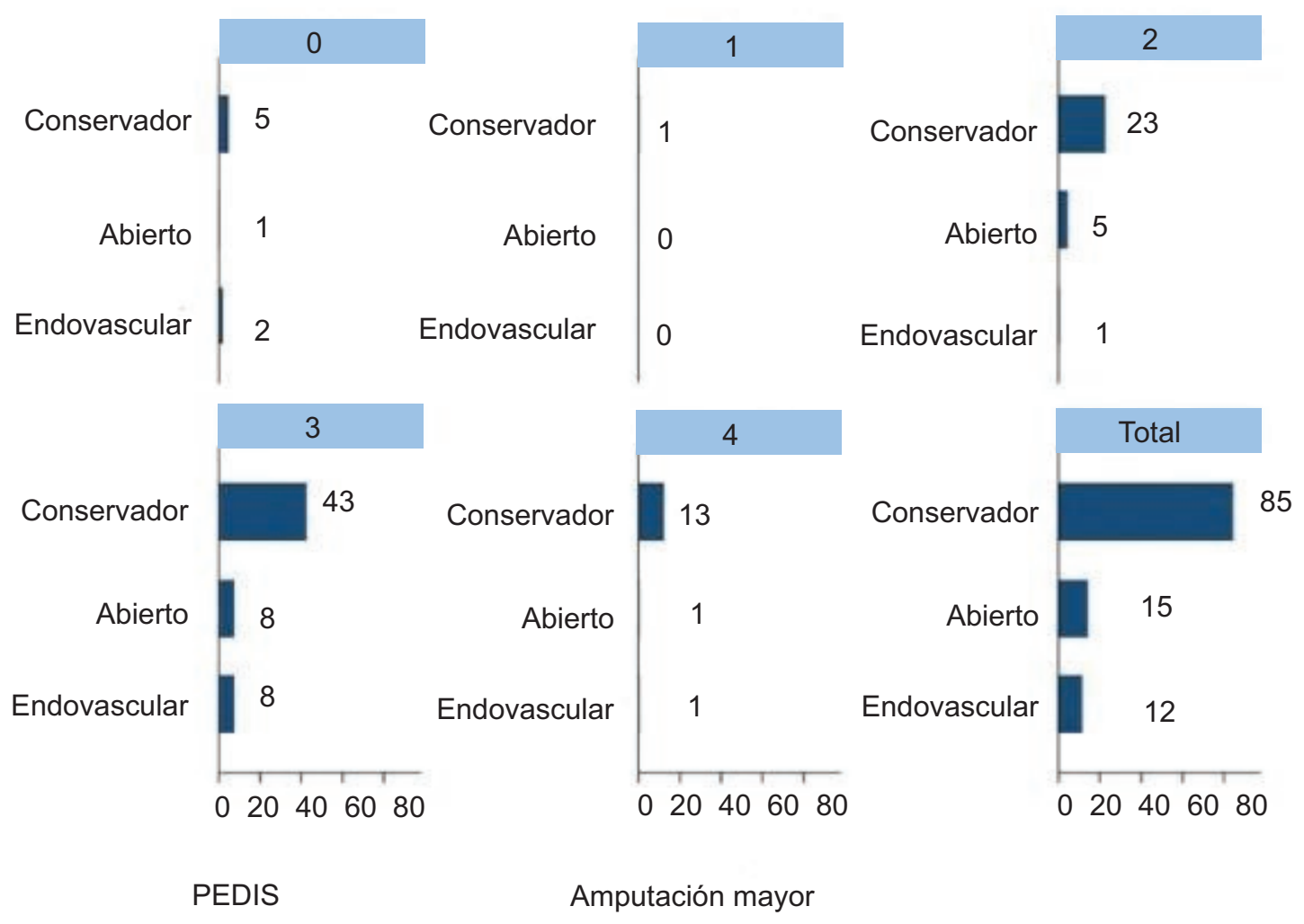

Figura 3. Distribución de los pacientes con amputación mayor que no recibieron revascularización y de los sometidos a revascularización (endovascular o abierta) de acuerdo con la clasificación PEDIS.

Tabla 2. Variables demográficas y comorbilidades de los pacientes sometidos a revascularización

\begin{tabular}{|c|c|c|c|c|}
\hline & $\begin{array}{l}\text { Salvamento de extremidad } \\
(\%)\end{array}$ & $\begin{array}{c}\text { Amputación mayor } \\
(\%)\end{array}$ & $\begin{array}{l}\text { Total } \\
\text { (n) }\end{array}$ & $\mathrm{p}$ \\
\hline Sexo & $\begin{array}{l}F: 48 \\
M: 50\end{array}$ & $\begin{array}{l}F: 52 \\
M: 50\end{array}$ & $\begin{array}{l}F: 33 \\
M: 20\end{array}$ & 1.00 \\
\hline HAS & 51 & 49 & 41 & 0.50 \\
\hline Obesidad & 67 & 33 & 3 & 1.00 \\
\hline Dislipidemia & 44 & 56 & 32 & 0.40 \\
\hline IAM & 31 & 69 & 16 & 0.13 \\
\hline ICC & 71 & 29 & 7 & 0.25 \\
\hline EAP & 50 & 50 & 42 & 1.00 \\
\hline EVC & 63 & 38 & 8 & 0.46 \\
\hline Hemodiálisis & 50 & 50 & 4 & 1.00 \\
\hline EPOC & 50 & 50 & 2 & 1.00 \\
\hline Estatinas & 56 & 44 & 18 & 0.56 \\
\hline Antiagregante & 49 & 51 & 35 & 1.00 \\
\hline Anticoagulante & 50 & 50 & 6 & 1.00 \\
\hline Osteomielitis & 50 & 50 & 28 & 0.78 \\
\hline Tabaco & 45 & 55 & 29 & 0.57 \\
\hline
\end{tabular}

F, femenino; M, masculino; HAS, hipertensión arterial sistémica, IAM, infarto agudo de miocardio; ICC, insuficiencia cardiaca congestiva; EAP, enfermedad arterial periférica; 

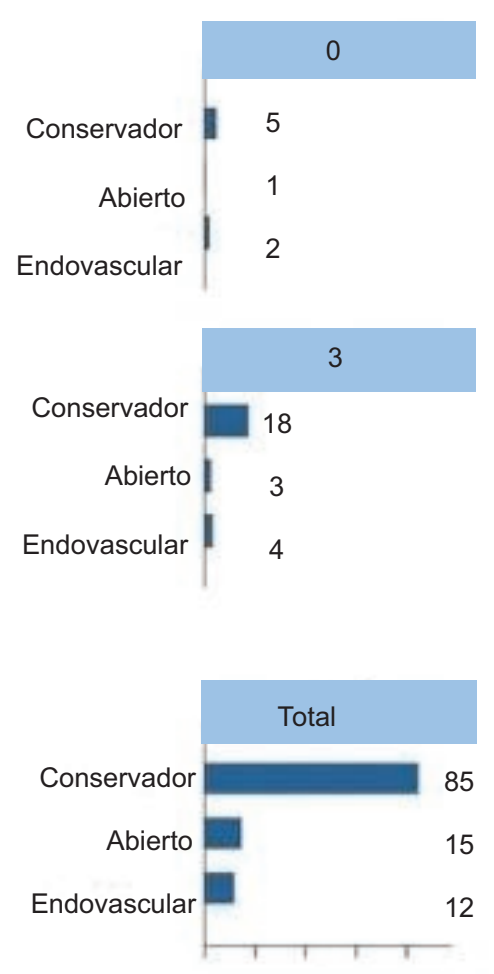

020406080
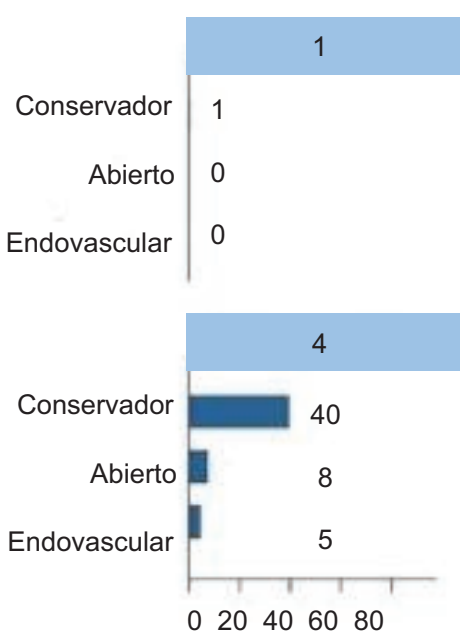

020406080
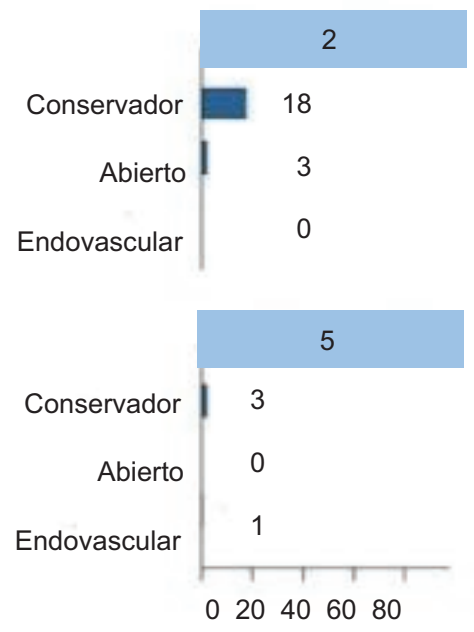

Wagner

Amputación mayor

Figura 4. Distribución de los pacientes con amputación mayor que no recibieron revascularización y de los sometidos a revascularización (endovascular o abierta) de acuerdo con la clasificación de Wagner.

Como se ha señalado, pocas investigaciones han examinado profundamente los factores que influyen en el resultado de la cicatrización de úlceras en pie diabético en el momento de la revascularización. ${ }^{20}$ En México, esto representa un problema de gran importancia debido a la alta prevalencia de diabetes mellitus, aunque algunos investigadores nacionales han sostenido que el salvamento de extremidad no está necesariamente ligado a la necesidad de revascularización. ${ }^{21}$ Por otra parte, la Sociedad Europea de Cirugía Vascular recomienda desbridamiento local en el sitio de la infección antes de un procedimiento de revascularización. ${ }^{22}$ De igual forma, en el Consenso TASC II (TransAtlantic Inter-Society Consensus) para el manejo de la enfermedad arterial periférica se indica que la revascularización debe considerarse si existen claros signos clínicos de isquemia crítica presente o si la cicatrización no ocurre a pesar de un óptimo tratamiento no invasivo. ${ }^{8}$

Aunque todavía existe información limitada sobre el resultado de la revascularización en pacientes con diabetes y enfermedad arterial periférica con una úlcera y la mayoría de los estudios que incluyen pacientes con y sin diabetes reportan un desenlace en términos de salvamento de extremidad en lugar de cicatrización de la úlcera, ${ }^{23}$ Faglia et al.$^{24}$ concluyeron respecto al desbridamiento temprano y la revascularización en pacientes con infección aguda profunda, que la revascularización temprana tenía mejores resultados que solo la observación clínica y el tratamiento antibiótico intravenoso.

Las limitaciones que reconocemos en nuestra investigación incluyen la naturaleza retrospectiva, un número reducido de pacientes y un potencial negativo de sesgo en la selección de quienes fueron sometidos a revascularización; en torno a este aspecto, la decisión de intervención fue de cirujanos vasculares independientes. La angioplastia con balón fue la opción inicial de tratamiento si se encontraba disponible, mientras que la revascularización abierta se realizó en pacientes no candidatos para angioplastia debido a causas clínicas o económicas. 
Tabla 3. Variables demográficas y comorbilidades de los pacientes no sometidos a revascularización

\begin{tabular}{|c|c|c|c|c|}
\hline & $\begin{array}{l}\text { Salvamento de extremidad } \\
(\%)\end{array}$ & $\begin{array}{c}\text { Amputación mayor } \\
(\%)\end{array}$ & $\begin{array}{c}\text { Total } \\
\text { (n) }\end{array}$ & $p$ \\
\hline Sexo & $\begin{array}{l}\text { F: } 64 \\
\text { M: } 66\end{array}$ & $\begin{array}{l}\text { F: } 36 \\
\text { M: } 34\end{array}$ & $\begin{array}{l}\text { F: } 89 \\
\text { M: } 165\end{array}$ & 0.78 \\
\hline HAS & 65 & 35 & 185 & 1.00 \\
\hline Obesidad & 73 & 27 & 52 & 0.193 \\
\hline Dislipidemia & 65 & 35 & 168 & 0.89 \\
\hline IAM & 42 & 58 & 38 & 0.003 \\
\hline ICC & 58 & 42 & 24 & 0.50 \\
\hline EAP & 44 & 56 & 72 & 0.001 \\
\hline EVC & 65 & 35 & 26 & 1.00 \\
\hline Hemodiálisis & 55 & 45 & 31 & 0.25 \\
\hline EPOC & 33 & 67 & 3 & 0.28 \\
\hline Estatinas & 57 & 43 & 84 & 0.70 \\
\hline Antiagregante & 55 & 45 & 130 & 0.001 \\
\hline Anticoagulante & 58 & 43 & 7 & 0.69 \\
\hline Osteomielitis & 62 & 38 & 129 & 0.28 \\
\hline Tabaco & 68 & 32 & 124 & 0.50 \\
\hline
\end{tabular}

F, femenino, M, masculino; IAM, infarto agudo de miocardio; ICC, insuficiencia cardiaca congestiva; EVC, evento vascular cerebral; EPOC, enfermedad pulmonar obstructiva crónica

En conclusión, observamos que menos de la mitad de los pacientes con pérdida de tejido avanzado de acuerdo con las clasificaciones PEDIS y de Wagner salvaron la extremidad a pesar de la revascularización. Una referencia temprana a los servicios de cirugía vascular y la implementación y evaluación de criterios de selección más apropiados, como el sistema de clasificación de Wound, Ischemia, and foot Infection, podría incrementar el salvamento de extremidades en este grupo de pacientes. El presente estudio hace énfasis en la necesidad de investigación referente a la selección de pacientes diabéticos con úlceras que podrían beneficiarse de la revascularización, abierta o endovascular, con el objetivo de salvar la extremidad.

\section{Declaración de intereses}

Los autores declaran que no tienen conflictos de intereses.

\section{Bibliografía}

1. Richard JL, Schuldiner S. Epidemiology of diabetic foot problems. Rev Med Int. 2008;29(Suppl 2):S222-S230.

2. Singh N, Armstrong DG, Lipsky BA. Preventing foot ulcers in patients with diabetes. JAMA. 2005;293(2):217-228.
3. Driver VR, Fabbi M, Lavery LA, Gibbons G. The cost of diabetic foot, the economic case for the limb salvage team. J Vasc Surg. 2010;52(3 Suppl):17S-22S.

4. Lavery LA, Armstrong DG, Wunderlich RP, Mohler MJ, Wendel CS, Lipsky BA. Risk factors for foot infections in individuals with diabetes. Diabetes Care. 2006;29(6):1288-1293.

5. Armstrong DG, Mills JL. Toward a change in syntax in diabetic foot care prevention, equals remission. $\mathrm{J}$ Am Podiatr Med Assoc. 2013;103(2):161-162.

6. Armstrong DG, Wrobel J, Robbins JM. Guest editorial: are diabetes related wounds and amputations worse than cancer? Int Wound J. 2007;4(4):286-287.

7. Apelqvist J, Bakker K, Van-Houtum WH, Nabuurs-Franssen $\mathrm{MH}$, Schaper NC. International consensus and practical guidelines on the management and the prevention of the diabetic foot. International Working Group on the Diabetic Foot. Diabetes Metab Res Rev. 2000;16(Suppl 1):S84-92.

8. Schaper NC, Apelvist J, Bakker K. Reducing lower leg amputation in diabetes: a challenge for patients healthcare providers and healthcare system. Diabetologia. 2012;55(7):1869-1872.

9. Armstrong DG, Lavery LA, Harkless LB. Validation of a diabetic wound classification system. The contributions of depth, infection, and ischemia to risk of amputation. Diabetes Care. 1998;21(5):855-859

10. Norgren L, Hiatt WR, Dormandy JA, Nehler MR, Harris KA, Fowkes FG. Inter-Society Consensus for the Management of Peripheral Arterial Disease (TASC II). J Vasc Surg. 2007;45(1 Suppl):S5-S67.

11. Eslami MH, Zayaruzny M, Fitzgerald GA. The adverse effects of race, insurance status, and low income on the rate of amputation in patients presenting with lower extremity ischemia. J Vasc Surg. 2007; 45(1):55-59.

12. Alexandrescu V, Hubermont G, Philips $Y$, Guillaumie B, Ngongang $C$, Coessens $\mathrm{V}$, et al. Combined primary subintimal and endoluminal angioplasty for ischaemic inferior limb ulcers in diabetic patients: 5-year practice in multidisciplinary diabetic foot service. Eur J Vasc Endovasc Surg. 2009;37(4):448-456.

13. Castro G, Liceaga G, Arrioja A, Calleja JM, Espejel A, Flores J, et al. Guía clínica basada en evidencia para el manejo del pie diabético. Med Int Mex. 2009;25(6):481-526.

14. Schaper NC. Diabetic foot ulcer classification system for research purposes: a progress report on criteria for including patients in research studies. Diabetes Metab Res Rev. 2004;20(Suppl 1):S90-S95. 
15. Bell PRF, Charlesworthd D, DePalma RG, Eastcostt HHG, Eklof B, Jamieson CW, et al. The definition of critical ischemia of a limb. Working Party of the International Vascular Symposium. Br J Surg. 1982; 69(Suppl 1):S2.

16. Apelquvist J, Elgzyri T, Larrson J, Löndahl M, Nyberg P, Thörne J. Factors related to outcome of neuroischemic/ischemic foot ulcer in diabetic patients. J Vasc Surg. 2011;53(6):1582-1588.

17. Wagner FW. The dysvascular foot: a system for diagnosis and treatment. Foot Ankle. 1981;2(2):64-122.

18. Mills JL Sr, Conte MS, Armstrong DG, Pomposelli FB, Schanzer A, Sidawy AN, et al. The Society for Vascular Surgery Lower Extremity Threatened Limb Classification System: risk stratification based on wound, ischemia, and foot infection (WIfl). J Vasc Surg. 2014;59(1):220234.e1-2

19. Zhan LX, Branco BC, Armstrong DG, Mills JL. The Society for Vascular Surgery lower extremity threatened limb classification system based on Wound, Ischemia, and foot Infection (WIfl) correlates with risk of major amputation and time to wound healing. J Vasc Surg. 2015;61(4):939-944.
20. Elgzyri T, Larsson J, Nyberg P, Thörne J, Eriksson KF, Apelqvist J. Early revascularization after admittance to a diabetic foot center affects the healing probability of ischemic foot ulcer in patients with diabetes. See comment in PubMed Commons belowEur J Vasc Endovasc Surg. 2014;48(4):440-446.

21. Cabeza-De-Vaca FG, Macias AE, Ramirez WA, Munoz JM, Alvarez JA Mosqueda JL, et al. Salvaging diabetic foot through debridement, pressure alleviation, metabolic control, and antibiotics. Wound Repair Regen. 2010;18(6):567-571.

22. Lepäntalo M, Apelqvist J, Setacci C, Ricco JB, De-Donato G, Becker F et al. Chapter V: diabetic foot. Eur J Vasc Endovasc Surg. 2011;42(Suppl. 2):S60-S74

23. Hinchliffe RJ, Andros G, Apelvist J, Bakker K, Friederich S, Lammer J, et al. A systemic review of the effectiveness of revascularization of the ulcerated foot in patients with diabetes and peripheral arterial disease. Diabetes Metab Res Rev. 2012;28(Suppl 1):179-217.

24. Faglia E, Clerici G, Caminiti M, Quaranteiello A, Gino M, Morabito A. The role of early surgical debridment and revascularization in patients with diabetes and deep foot space abscess: retrospective review of 106 patients with diabetes. J Foot Ankle Surg. 2006;45(4):220-226. 